\title{
Liver Resection for Hepatocellular Carcinoma in Patients with Renal Dysfunction
}

\author{
Chikara Shirata $^{1} \cdot$ Kiyoshi Hasegawa $^{1} \cdot$ Takashi Kokudo $^{1} \cdot$ Suguru Yamashita $^{1} \cdot$ \\ Satoshi Yamamoto ${ }^{1} \cdot$ Junichi Arita $^{1} \cdot$ Nobuhisa Akamatsu $^{1} \cdot$ Junichi Kaneko $^{1}$. \\ Yoshihiro Sakamoto $^{1} \cdot$ Norihiro Kokudo $^{1,2}$
}

Published online: 8 June 2018

(C) Société Internationale de Chirurgie 2018

\begin{abstract}
Background The aim of this study was to evaluate the feasibility of liver resection in hepatocellular carcinoma (HCC) patients with preoperative renal dysfunction (RD).

Methods Data from 735 patients undergoing primary liver resection for HCC between 2002 and 2014 were analyzed. Short- and long-term outcomes were compared between the RD group, defined by a preoperative estimated glomerular filtration rate of $<45 \mathrm{~mL} / \mathrm{min} / 1.73 \mathrm{~m}^{2}$, and the non-RD group.

Results Sixty-two patients had RD. The incidence of postoperative pleural effusion (24 vs. $11 \% ; P=0.007)$ and major complications (Clavien-Dindo III-V; 31 vs. $15 \% ; P=0.003$ ) were significantly higher in RD patients. In RD patients with Child-Pugh A, 90-day mortality rate $(1.9 \%)$ and median survival time (6.11 years) were comparable to that of non-RD patients. In contrast, RD patients with Child-Pugh B had a very high 90 -day mortality rate (22.2\%), and a significant shorter median survival time compared to non-RD patients (1.19 vs. 4.84 years; $P=0.001)$.

Conclusions Liver resection for Child-Pugh A patients with RD is safe and has comparable oncological outcomes compared to non-RD patients. However, selection of liver resection candidates from Child-Pugh B patients with RD should be stricter.
\end{abstract}

\section{Abbreviations}

RD Renal dysfunction

HCC Hepatocellular carcinoma

eGFR Estimated glomerular filtration rate

CT Computed tomography

PHT Portal hypertension

Electronic supplementary material The online version of this article (https://doi.org/10.1007/s00268-018-4698-3) contains supplementary material, which is available to authorized users.

Norihiro Kokudo

nkokudo@hosp.ncgm.go.jp

1 Hepato-Biliary-Pancreatic Surgery Division, Department of Surgery, Graduate School of Medicine, The University of Tokyo, 7-3-1 Hongo, Bunkyo-ku, Tokyo 113-8655, Japan

2 National Center for Global Health and Medicine, 1-21-1 Toyama, Shinjuku-ku, Tokyo 162-8655, Japan
MST Median survival time

OS Overall survival

\section{Introduction}

With the recent advances in surgical techniques and perioperative management techniques, liver resection has become a safe surgical procedure with a low mortality rate [1-3]. Furthermore, liver resection is now performed in patients with preoperative comorbidities. Renal dysfunction (RD) has been reported as a risk factor for the development of postoperative complications, such as massive ascites, respiratory failure, or acute renal failure, in patients undergoing hepatic resection [4-7]. Another concern in $\mathrm{RD}$ patients is the risk of needing postoperative 
initiation of hemodialysis. Until now, only one study [6] has reported liver resection in preoperative RD patients not requiring hemodialysis postoperatively.

The prognosis of patients with preoperative RD following resection has remained controversial [4-7]. While Toshima et al. [6] and Kaibori et al. [4] have reported that preoperative RD has no influence on long-term outcomes after liver resection, Orii et al. [5] demonstrated a significantly worse overall survival (OS) in RD compared to nonRD subjects.

Currently, little is known about the impact of preoperative RD on the short- and long-term outcomes of hepatic resection in patients with hepatocellular carcinoma (HCC). The aim of the present study was to establish a set of criterion for surgical indication for HCC patients with preoperative RD.

\section{Materials and methods}

This study was conducted with the approval of the Institutional Ethics Review Board of the University of Tokyo (No. 2158). Written informed consent was obtained from all patients.

We enrolled 735 HCC patients who had undergone liver resection for the first time at a tertiary care hospital in Japan between October 2002 and July 2014. Of them, 62 had preoperative RD. Disease progression and resectability statuses were assessed by imaging studies, including contrast-enhanced computed tomography (CT), magnetic resonance imaging, and ultrasonography. Liver function impairment caused by an underlying liver disease was assessed by liver function testing, evaluation of Child-Pugh class, and estimation of the indocyanine green retention rate at $15 \mathrm{~min}$ [8]. Preoperative ascites was considered a contraindication to surgical resection. There was no patient with a history of hepatorenal syndrome. When pleural effusion was suspected by routine chest X-ray postoperatively, ultrasound examination was performed, and paracentesis was performed when an effusion was detected. A repeat paracentesis was performed by the same manner when necessary. The chest tube was removed when the amount of discharge decreased to less than $200 \mathrm{~mL} /$ day. To evaluate the feasibility of liver resection in $\mathrm{HCC}$ patients with RD, the primary endpoint of our study was short-term outcomes (postoperative complications and 90-day mortality) after hepatectomy. The secondary endpoint was long-term outcomes [median survival time (MST)].

\section{Diagnostic criteria for $\mathrm{RD}$}

Preoperative RD was defined by a preoperative estimated glomerular filtration rate (eGFR) of $<45 \mathrm{~mL} / \mathrm{min} / 1.73 \mathrm{~m}^{2}$ and chronic kidney disease [9] Stage 3B or higher; Stage 3B chronic kidney disease is reportedly associated with the sharpest rise in the overall risk profile [10-12]. Non-RD was defined as an eGFR $\geq 45 \mathrm{~mL} / \mathrm{min} / 1.73 \mathrm{~m}^{2}$.

Major liver resection was defined as resection of 3 or more Couinaud's segments. Portal hypertension (PHT) was diagnosed on the basis of the presence of esophageal varices or splenomegaly associated with a platelet count of $<10 \times 10^{4}$ cells/mL [13-15]. Postoperative complications were recorded and graded according to the Clavien-Dindo classification system [16]. Pleural effusions treated by diuretics were considered as Grade II, while pleural effusion treated by paracentesis was considered as Grade IIIa. Postoperative mortality was defined as death within 90 days of surgery.

\section{Perioperative management of patients with RD}

Application of criteria for liver resection and selection of surgical procedures were made regardless of renal function. All RD patients were referred to the nephrology team. Hemodialysis was performed immediately when the postoperative serum potassium level was $>6 \mathrm{mEq} / \mathrm{L}$. For patients on maintenance hemodialysis, the hemodialysis was scheduled to be performed the day before surgery, postoperative day 1 or 2 , and then three times per week thereafter. If pulmonary edema was suspected, continuous hemodiafiltration was immediately performed. Potassium canrenoate was routinely administered to reduce ascitic fluid drainage in patients with normal renal function [3, 17]; however, this drug was administered with caution in $\mathrm{RD}$ patients while checking the serum potassium or creatinine level to avoid hyperkalemia or exacerbation of renal function. The ascitic fluid drainage volume was measured daily. Patients with normal renal function and RD patients not requiring maintenance hemodialysis received a fluid infusion of $40 \mathrm{~mL} / \mathrm{kg} /$ day (patients with liver cirrhosis) or $45 \mathrm{~mL} / \mathrm{kg} /$ day (patients without liver cirrhosis) [3, 17]. The total fluid infusion volume was restricted as follows in patients under hemodialysis to avoid pulmonary edema: total fluid infusion = urinary output + ascitic fluid drainage and pleural effusion aspirated volumes + insensible water loss. Ascitic fluid collection included discharge from the thoracic tube, abdominal tube, and gauze oozing. Insensible water loss was calculated as $12 \mathrm{~mL} / \mathrm{kg} /$ day.

\section{Statistical analysis}

Statistical analyses were performed using JMP 11 software (SAS Institute Inc., Cary, NC, USA). Categorical variables were analyzed by the Chi-square or Fisher's exact test, as appropriate. Continuous variables were analyzed using the 
Mann-Whitney $\mathrm{U}$ test; $P<0.05$ was considered statistically significant. Overall survival curves were determined using the Kaplan-Meier method and compared using the log-rank test. A multivariate analysis was performed using a logistic regression and backward elimination procedure; $P<0.10$ was set as the cut-off value for elimination. Because we used the backward elimination procedure, the multivariate analysis was independently executed. The following 10 variables were examined as potential risk factors for postoperative pleural effusion and major complication: a positive serum test for the hepatitis $\mathrm{C}$ virus antibody, Child-Pugh class B, platelet count $<10 \times 10^{4}$ cells $/ \mu \mathrm{L}, 15$-min indocyanine green retention rate $>15 \%$, liver cirrhosis, blood loss $>500 \mathrm{~mL}$, operative time $>300 \mathrm{~min}$, intraoperative red blood cell transfusion, serum albumin $<3.5 \mathrm{~g} / \mathrm{dL}$, and eGFR $<45 \mathrm{~mL} / \mathrm{min} / 1.73 \mathrm{~m}^{2}$. We performed a 1:1 match using logit (propensity score) through the nearest available matching to evaluate the effect of comorbidities (diabetes mellitus and cardiovascular disease) on postoperative outcomes. Propensity scores were created using logistic regression model.

\section{Results}

\section{Patient characteristics}

Sixty-two patients had RD, and 16 received routine preoperative hemodialysis. Diabetic nephropathy (33 patients, $53 \%$ ) was the most frequent cause of preoperative RD in the study participants, followed by hypertensive nephropathy (nine patients, $15 \%$ ), glomerulonephritis (four patients, 6.5\%), post hemi-nephrectomy (four patients, 6.5\%), drug induced nephritis (two patients, 3.2\%), and IgA nephropathy (two patients, 3.2\%). The preoperative characteristics of non-RD and $\mathrm{RD}$ patient groups are summarized in Table 1. The median age (70 vs. 67 years; $P=0.0005)$, the incidence of diabetes mellitus (30 vs. $65 \% ; P<0.001)$ and cardiovascular disease (11 vs. $37 \%$; $P<0.001)$ in RD patients were significantly higher than in non-RD patients. Tumor characteristics are shown in Table 2. There were no significant differences in tumor characteristics between RD and non-RD patients.

\section{Intraoperative characteristics of non-RD and RD groups}

As listed in Table 3, intraoperative characteristics were comparable for all patients, except for blood loss and the percentage of patients requiring red blood cell transfusion that were higher in the RD group.

\section{Short-term outcomes}

The incidence of pleural effusion and a major complication was significantly higher in the RD than in the non-RD group (Table 3 ). The median postoperative hospital stay was not significantly different between the groups. There was no mortality during surgery or within 30 days after surgery. Three cases in each RD and non-RD group died within 90 days after surgery. In the non-RD group, two patients died due to the rapid recurrence of tumor and one due to cholangitis. In the RD group, two patients died due to the rapid recurrence of tumor, while one due to pneumonia. Two patients in each group, including the 90-day mortality evaluation, died during hospitalization. Figure 1 shows the incidence of pleural effusion (Fig. 1a) and 90-day mortality rate (Fig. 1b) according to renal function and Child-Pugh class. A remarkably high incidence of pleural effusion and mortality rate were observed in RD and Child-Pugh B patients. The median eGFR values in Child-Pugh A patients with RD decreased from postoperative day $7\left(37.8 \mathrm{~mL} / \mathrm{min} / 1.73 \mathrm{~m}^{2}\right)$ to days $14(33.1 \mathrm{~mL} /$ $\min / 1.73 \mathrm{~m}^{2}$ ) and recovered to a preoperative level $\left(36.6 \mathrm{~mL} / \mathrm{min} / 1.73 \mathrm{~m}^{2}\right)$ between days $30(35.3 \mathrm{~mL} / \mathrm{min} /$ $\left.1.73 \mathrm{~m}^{2}\right)$ and $90\left(37.4 \mathrm{~mL} / \mathrm{min} / 1.73 \mathrm{~m}^{2}\right)$. None of the Child-Pugh A patients with RD developed postoperative acute kidney failure requiring emergent hemodialysis. In RD with Child-Pugh B group, two patients needed hemodialysis postoperatively. Univariate analysis of pleural effusion is shown in Supplementary Table 1. Multivariate analysis carried out to determine risk factors for postoperative pleural effusion identified an eGFR $<45 \mathrm{~mL} /$ $\mathrm{min} / 1.73 \mathrm{~m}^{2}$ (risk ratio, 2.55; 95\% confidence interval, $1.32-4.70 ; P=0.0063$ ) as significant independent risk factors (Table 4). We also performed univariate (Supplementary Table 1) and multivariate analyses (Table 4) for major complication. Blood loss $>500 \mathrm{~mL}$, an eGFR $<45 \mathrm{~mL} / \mathrm{min} / 1.73 \mathrm{~m}^{2}$, and hepatitis $\mathrm{C}$ virus infection were found to be the independent risk factors for major complication in multivariate analysis.

\section{Long-term outcomes}

The median follow-up time was 5.07 (95\% CI 4.60-5.42) years. During the follow-up period, 25 of the $62 \mathrm{RD}$ patients died. The causes of death were cancer $(n=17)$, cerebrovascular disease $(n=3)$, acute myocardial infarction $(n=2)$, pneumonia $(n=1)$, ARDS $(n=1)$, and a traffic accident $(n=1)$. There was no death related to liver failure. MST was 5.48 years in the RD group and 7.66 years in the non-RD group, with the difference marginally significant $(P=0.059$; Fig. 2a). MST for Child-Pugh A patients (9.30 years) were significantly higher than that of ChildPugh B patients (4.63 years; $P<0.001$; Fig. $2 b$ ). In Child- 
Table 1 Preoperative characteristics of patients with or without renal dysfunction (RD)

\begin{tabular}{|c|c|c|c|}
\hline & $\begin{array}{l}\text { Non-RD } \\
N=673(92 \%)\end{array}$ & $\begin{array}{l}\mathrm{RD} \\
N=62(8 \%)\end{array}$ & $P$ values \\
\hline Age (y) median (IQR) & $67(58-73)$ & $70(65-76)$ & $<0.001$ \\
\hline Gender (male/female) & $537 / 136(80 / 20)$ & $45 / 17(73 / 27)$ & 0.192 \\
\hline Body Mass Index $\left(\mathrm{kg} / \mathrm{m}^{2}\right)$ median (IQR) & $22.9(21.1-25.0)$ & $22.9(21.5-24.9)$ & 0.962 \\
\hline Diabetes mellitus & $199(30)$ & $40(65)$ & $<0.001$ \\
\hline Cardiovascular disease $^{\mathrm{a}}$ & $71(11)$ & $23(37)$ & $<0.001$ \\
\hline \multicolumn{4}{|l|}{ Laboratory data (median, IQR) } \\
\hline Hemoglobin (g/dl) & $13.2(12.2-14.2)$ & $11.4(10.3-13.2)$ & $<0.001$ \\
\hline Albumin $(\mathrm{g} / \mathrm{dl})$ & $3.8(3.5-4.1)$ & $3.7(3.4-3.9)$ & 0.094 \\
\hline Prothrombin time $(\%)$ & $88(76-100)$ & $100(83-100)$ & 0.003 \\
\hline \multicolumn{4}{|l|}{ Underlying liver diseases } \\
\hline None & $61(9.2)$ & $7(11)$ & 0.498 \\
\hline Chronic hepatitis/liver fibrosis & $346(52)$ & $38(62)$ & 0.143 \\
\hline Liver cirrhosis & $254(38)$ & $16(26)$ & 0.072 \\
\hline \multicolumn{4}{|l|}{ Etiology of hepatic diseases } \\
\hline HBV & $161(24)$ & $11(18)$ & 0.347 \\
\hline $\mathrm{HCV}$ & $328(49)$ & $32(52)$ & 0.692 \\
\hline Alcoholic & $203(30)$ & $22(35)$ & 0.390 \\
\hline ICGR-15 (\%) median (IQR) & $12.6(8.5-19)$ & $9.7(6.4-17)$ & 0.031 \\
\hline Child-Pugh grade A/B/C (\%) & 613/59/1 (91/8.8/0.2) & $53 / 9 / 0(85 / 15 / 0)$ & 0.235 \\
\hline MELD score median (IQR) & $6.9(2.7-8.7)$ & $11.1(6.0-17)$ & $<0.001$ \\
\hline
\end{tabular}

$I Q R$ interquartile range, $H B V$ hepatitis B virus, $H C V$ hepatitis $\mathrm{C}$ virus, ICGR-15 indocyanine green retention rate at 15 min, $M E L D$ model for end-stage disease

${ }^{\mathrm{a}}$ Exclusion: hypertension only

Table 2 Tumor characteristics of patients with or without renal dysfunction (RD)

\begin{tabular}{|c|c|c|c|}
\hline & $\begin{array}{l}\text { Non-RD } \\
N=673(92 \%)\end{array}$ & $\begin{array}{l}\mathrm{RD} \\
N=62(8 \%)\end{array}$ & $P$ values \\
\hline Tumor size (mm) median (IQR) & $36(23-60)$ & $39(27-60)$ & 0.461 \\
\hline Major vessel invasion & $67(10)$ & $7(11)$ & 0.826 \\
\hline Intrahepatic metastases & $104(15)$ & $8(13)$ & 0.836 \\
\hline Positive margin & $5(0.7)$ & $1(1.6)$ & 0.345 \\
\hline \multicolumn{4}{|l|}{ Pathological grade } \\
\hline Well differentiated & $169(26)$ & $11(18)$ & 0.219 \\
\hline Moderate to poor differentiated & $492(74)$ & $51(82)$ & \\
\hline Tumors number median (IQR) & $1(1-2)$ & $1(1-2)$ & 0.607 \\
\hline Extra hepatic metastases & $25(3.7)$ & $3(4.8)$ & 0.421 \\
\hline UICC TNM stage & & & 0.469 \\
\hline I & $351(54)$ & $32(52)$ & \\
\hline II & $181(28)$ & $14(23)$ & \\
\hline III & $97(15)$ & $12(19)$ & \\
\hline IV & $26(4.0)$ & $4(6.5)$ & \\
\hline
\end{tabular}

$I Q R$ interquartile range, UICC union for international cancer control

Pugh A patients, the OS was similar between RD (MST, 6.11 years) and non-RD groups (MST, 9.74 years; $P=0.514$; Fig. 2c). On the other hand, in Child-Pugh B patients, the $\mathrm{OS}$ in the RD group was significantly worse than that in the non-RD group (MST, 1.19 vs. 4.84 years; $P=0.001$; Fig. 2 d). The median recurrence-free survival time did not differ between RD patients and non-RD patients [1.62 years (CI 0.78-5.21 years) vs. 1.78 years (CI 
Table 3 Intraoperative and postoperative characteristics of patients with or without renal dysfunction (RD)

\begin{tabular}{|c|c|c|c|}
\hline & $\begin{array}{l}\text { Non-RD } \\
N=673(92 \%)\end{array}$ & $\begin{array}{l}\text { RD } \\
N=62(8 \%)\end{array}$ & $P$ values \\
\hline \multicolumn{4}{|l|}{ Intra-operative variables } \\
\hline Median operative time; min (IQR) & $358(275-460)$ & $357(281-459)$ & 0.486 \\
\hline Major liver resection $^{\mathrm{a}}$ & $161(24)$ & $17(27)$ & 0.537 \\
\hline Minor liver resection & $512(76)$ & $45(73)$ & \\
\hline Blood loss (mL) median (IQR) & $650(380-1160)$ & $900(535-1466)$ & 0.023 \\
\hline Pringle's maneuver (min) median (IQR) & $70(46-97)$ & $64(47-85)$ & 0.403 \\
\hline Laparoscopic surgery & $20(3.0)$ & $2(3.2)$ & 0.708 \\
\hline Required RBC transfusion & $66(10)$ & $18(30)$ & $<0.001$ \\
\hline Required FFP transfusion & $235(35)$ & $29(47)$ & 0.889 \\
\hline \multicolumn{4}{|l|}{ Postoperative variables } \\
\hline \multicolumn{4}{|l|}{ Complications $^{\mathrm{b}}$} \\
\hline Bile leakage & $28(4.2)$ & $3(4.8)$ & 0.740 \\
\hline Pleural effusion & $74(11)$ & $15(24)$ & 0.007 \\
\hline Ascites & $97(14)$ & $13(21)$ & 0.191 \\
\hline Major complication (Grade $\geq 3$ ) & $99(15)$ & $19(31)$ & 0.003 \\
\hline Minor complication (Grade 1-2) & $216(32)$ & $23(37)$ & 0.479 \\
\hline Median hospital stay; day (IQR) & $15(12-20)$ & $16(13-23)$ & 0.187 \\
\hline 90-day mortality & $3(0.5)$ & $3(4.8)$ & 0.010 \\
\hline
\end{tabular}

$I Q R$ interquartile range, $R B C$ red blood cells, $F F P$ fresh frozen plasma

${ }^{a}$ Major liver resection: 3 or more segments resection, Minor liver resection: 1 or 2 segments resection

${ }^{\mathrm{b}}$ Classified Grade II or higher in Dindo-Clavien classification

1.45-2.11 years), $P=0.589$ ] (Fig. 2e). We also investigated the effect of Model for End-Stage Liver Disease (MELD) score in RD patients (Supplementary Fig. 1a). OS was different between high MELD group (MELD score $\geq 10$ ) and low MELD group (MELD score $<10$ ) (MST: 3.72 vs. 3.94 years, $P=0.039$ ). However, a model based on Child-Pugh grade (Supplementary Fig. 1b) showed better discrimination of OS than a model based on the MELD score.

\section{Characteristics of hemodialysis and non- hemodialysis groups}

We evaluated the effect of preoperative routine hemodialysis in RD patients with Child-Pugh A. Of the 53 RD patients with Child-Pugh A, 16 (30.2\%) were under routine hemodialysis. There were no significant differences in postoperative outcomes and MST after resection between these two groups (Supplementary Table 2).

\section{Impact of portal hypertension in RD patients with Child-Pugh A}

In Child-Pugh A patients with $\mathrm{RD}$, six patients were diagnosed with PHT (three with splenomegaly with a platelet count of $<10 \times 10^{4}$ cells $/ \mathrm{mL}$, two with the presence of esophageal varices, and one with both). There were no significant differences in the incidence of postoperative pleural effusion (33 vs. $19 \% ; P=0.592$ ), major complications (33 vs. $19 \% ; P=0.592)$, and MST (6.32 vs. 5.69 years; $P=0.402)$ between RD patients with and without PHT in Child-Pugh A patients group.

\section{Impact of diabetes mellitus and cardiovascular disease}

Because the occurrence of preoperative diabetes mellitus and cardiovascular disease was significantly higher in the $\mathrm{RD}$ group, we performed propensity score matching using these covariates (Supplementary Table 3). After matching, the frequency of diabetes mellitus and cardiovascular disease was 65 and 37\%, respectively, in both groups. The rate of postoperative pleural effusion was higher in the RD (24\%) than in the non-RD group $(8.1 \% ; P=0.026)$. The rate of major complication and 90-day mortality were higher in the RD group; however, the difference was not significant. MST was 5.48 and 6.85 years in the RD and non-RD groups, respectively $(P=0.557)$. These findings were similar to those obtained before matching. 


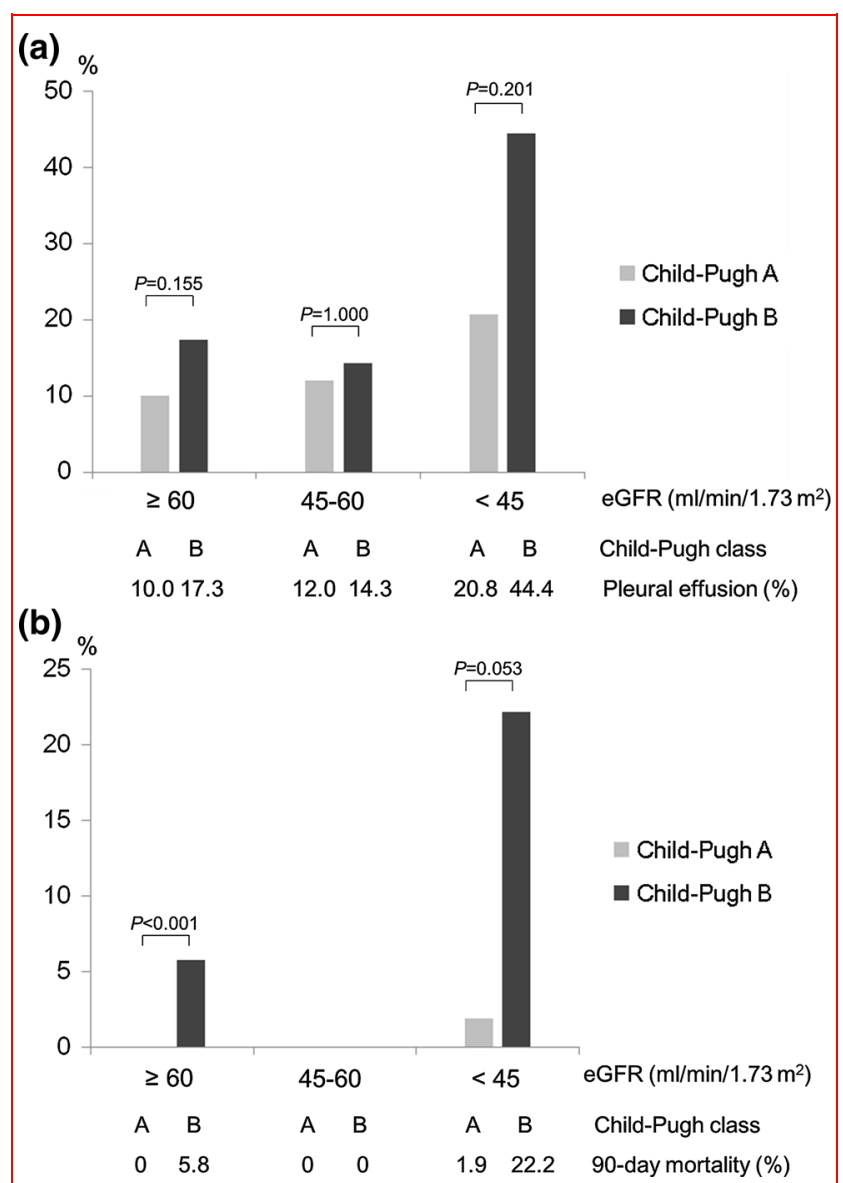

Fig. 1 Incidence of pleural effusion (a) and 90-day mortality rate (b) according to preoperative renal function and Child-Pugh class

Table 4 Risk factors for pleural effusion and major complication in multivariate analyses

$P$ value Odds ratio $(95 \%$ CI)

\begin{tabular}{lcc} 
Pleural effusion & & \\
eGFR $<45 \mathrm{~mL} / \mathrm{min} / 1.73 \mathrm{~m}^{2}$ & 0.0063 & $2.55(1.32-4.70)$ \\
$\begin{array}{l}\text { Operative time }>300 \mathrm{~min} \\
\text { Major complication }\end{array}$ & 0.1502 & $1.44(0.88-2.46)$ \\
$\begin{array}{l}\text { Blood loss }>500 \mathrm{~mL} \\
\text { eGFR }<45 \mathrm{~mL} / \mathrm{min} / 1.73 \mathrm{~m}^{2}\end{array}$ & $<0.001$ & $3.23(1.96-5.59)$ \\
$\begin{array}{l}\text { A positive serum test for the hepatitis C } \\
\quad \text { virus antibody }\end{array}$ & 0.0007 & $2.02(1.34-3.09)$ \\
\hline
\end{tabular}

$C I$ confidence interval, $e G F R$ estimated glomerular filtration rate

\section{Discussion}

The current study revealed that liver resection for ChildPugh A HCC patients with RD was safe and had comparable oncological outcomes compared to non-RD patients. However, selection of liver resection candidates from
Child-Pugh B HCC patients with RD should be more stringent.

In Child-Pugh A patients with RD, liver resection was associated with a relatively low 90-day mortality rate $(1.9 \%)$ and acceptable 5-year OS rate $(61.6 \%)$, with no significant difference in MST after surgery between RD and non-RD groups (6.11 vs. 9.74 years; $P=0.514$ ). Conversely, the prognosis of Child-Pugh B patients with $\mathrm{RD}$ was significantly worse than that of non-RD patients. In RD patients, OS in Child-Pugh B patients was significantly worse than in Child-Pugh A patients (MST, 6.11 vs. 1.19 years, $P<0.001$ ) (Supplementary Fig. 1 b). Moreover, 90-day morality was very high $(22.2 \%)$ in RD patients with Child-Pugh B. Thus, while liver resection is a good therapeutic choice for HCC patients with RD and Child-Pugh A, it is not the optimal therapeutic choice for RD patients with Child-Pugh B (Fig. 3).

Furthermore, we also assessed the impact of the type of resection (major or minor liver resection) and the presence of liver cirrhosis and found no significant difference in the short- and long-term outcomes (pleural effusion: 29 vs. $22 \%, P=0.740$; 90 -day mortality: 12 vs. $2.2 \%, P=0.180$; and MST: 2.70 vs. 5.69 years, $P=0.057$ ) between major and minor liver resections. Moreover, liver cirrhosis did not increase the postoperative risk (pleural effusion: 38 vs. $20 \%, P=0.118$; 90 -day mortality: 0 vs. $6.7 \%, P=0.560$; and MST: 5.69 vs. 4.79 years, $P=0.893$ ) compared with normal liver or chronic hepatitis. In RD patients, the liver function (Child-Pugh grade) was considered to be the principal factor. Because we selected the type of resection based on liver function in addition to the extent of disease progression [3], major liver resection was not opted in patients with poor liver function. This selection of the type of resection possibly affected the analysis of postoperative outcomes based on the type of resection. Although MELD score was reportedly to be useful in predicting postoperative outcomes after liver resection, a model based on the Child-Pugh grade showed better discrimination of OS than a model based on the MELD score in RD patients.

Postoperative renal function was well preserved in RD and Child-Pugh A patients, and none of them needed urgent postoperative hemodialysis. Only two RD patients with Child-Pugh B required urgent postoperative hemodialysis due to multiple organ failure caused by pneumonia or a rapid progression of recurrent cancer. Toshima et al. [6] reported that none of their patients with preoperative $\mathrm{RD}$ required urgent hemodialysis during hospitalization. Although Squires et al. [7] reported that preoperative renal insufficiency was associated with an increased risk of renal failure requiring initiation of dialysis, the results of Toshima et al., as well as our own, indicate that surgical resection may not induce acute renal failure necessitating urgent hemodialysis. The previously 


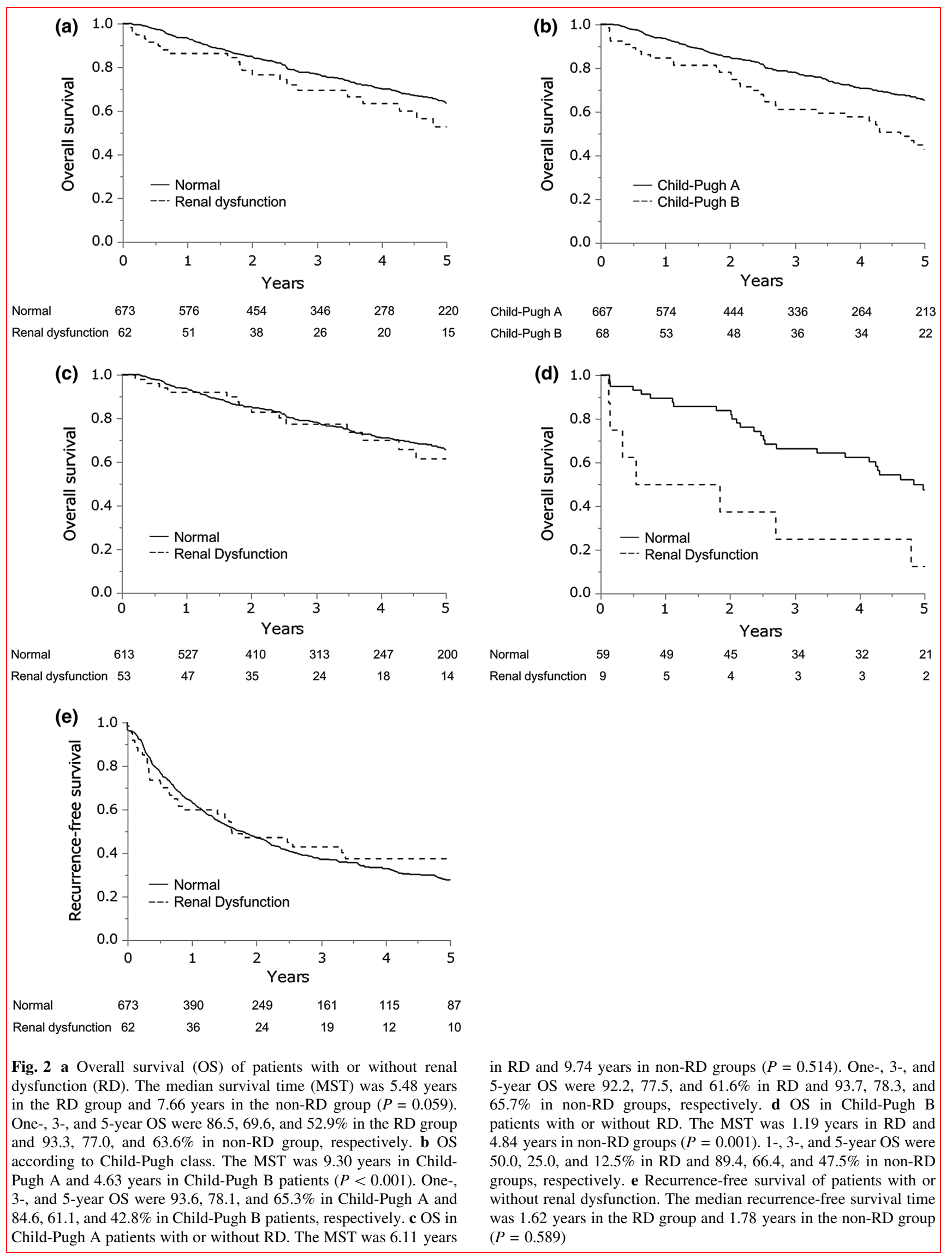






Fig. 3 Proposed surgical indication for hepatocellular carcinoma patients with preoperative renal dysfunction

reported median interval from the first diagnosis of RD to initiation of hemodialysis therapy is 6.1-14.2 months $[18,19]$. In RD patients with Child-Pugh A, postoperative hemodialysis was initiated in seven out of 37 cases who were not on preoperative hemodialysis. The median interval from surgery to initiation of hemodialysis was 38.5 months (IQR 16.0-63.2 months). These data suggest that liver resection may not affect the timing of initiation of hemodialysis.

Higher intraoperative median blood loss in RD patients is an additional problem $(900 \mathrm{~mL}$ in $\mathrm{RD}$ vs. $650 \mathrm{~mL}$ in non-RD group, $P=0.023$ ). In the RD group, 27 out of 62 RD patients $(44 \%)$ were treated by antiplatelet or anticoagulant drugs for comorbidities such as cardiovascular and cerebrovascular disease. Moreover, RD patients had a higher incidence of preoperative anemia than non-RD patients. Therefore, the intraoperative blood loss and red blood cell transfusion might have been higher in RD patients. To increase serum hemoglobin, administration of erythropoietin or transfusion might be considered preoperatively in RD patients.

$\mathrm{RD}$ was identified as a significant risk factor for postoperative pleural effusion. Nobili et al. [20] demonstrated four independent risk factors for the development of pleural effusion; however, they did not identify preoperative RD as a risk factor. This is probably due to the small number of RD patients in their study (11 patients) compared to ours (62 patients). Toshima et al. [6] demonstrated that $\mathrm{RD}$ was at an increased risk of massive ascites and pleural effusion. Our results were consistent with this report. All RD patients developing postoperative pleural effusion in our study required diuretics or paracentesis [12 patients $(80 \%)$ required paracentesis]. The incidence of pleural effusion did not differ significantly in patients with or without preoperative cardiovascular disease (11 vs. 12\%,
$P=0.736$ ). Although patients with pleural effusion had a longer median hospital stay ( 23 vs. 15 days, $P<0.001$ ), it was not considered that pleural effusion correlated to mortality.

Because the diagnosis of hepatorenal syndrome requires the presence of ascites [21], and surgery is contraindicated for patients with ascites at our institution, a major part of hepatorenal syndrome is estimated to be excluded. However, subclinical hepatorenal syndrome might exist. Although the rate of Child-Pugh B patients undergoing liver resection is higher in Japan (10.3\%) [22] compared to those in Western countries or China (3.9-6.4\%) [23-26], the present criteria provide universally useful information for surgical decision making in Child-Pugh B patients.

One of the limitations of the current study is its retrospective and single-institute design, and the relatively small number of patients with $\mathrm{RD}(n=62)$. The number of patients with RD was significantly smaller than patients without RD, and the results may have been biased because of limited statistical power. Furthermore, the number of Child-Pugh B patients with RD was extremely small $(n=9)$, which may have affected the statistical significance. Another limitation of this study is the low rate of laparoscopic liver resection (3.0\%), which reportedly decreases the rate of postoperative ascites and shortens hospital stay [27-29]. Thus, our proposal of surgical indication needs validation at centers where laparoscopic liver resections are more frequently performed. The impact of $\mathrm{RD}$ on outcomes after liver resection has been reported in eight studies [4-7, 30-33]. Of these, four studies deduced that RD itself was not a surgical contraindication, whereas one study suggested the need for careful patient selection. In the present study, we first revealed that preoperative RD had an important impact on the postoperative outcome, especially in Child-Pugh B patients. To the best of our knowledge, our study is the largest single-institution series of liver resections for HCC patients with RD and impaired liver function. Further studies are needed using large registry databases.

In conclusion, liver resection for Child-Pugh A HCC patients with RD is safe and has comparable oncological outcomes compared to non-RD patients. However, selection of liver resection candidates from Child-Pugh class B HCC patients with RD should be stricter.

Acknowledgements This work was supported by a grants-in-aid of The Public Trust Fund For Clinical Cancer Research.

\section{Compliance with ethical standards}

Conflict of interest The authors have no conflicts of interest to declare. 


\section{References}

1. Torzilli G, Makuuchi M, Inoue K et al (1999) No-mortality liver resection for hepatocellular carcinoma in cirrhotic and noncirrhotic patients: is there a way? A prospective analysis of our approach. Arch Surg 134:984-992

2. Fan ST, Lo CM, Liu CL et al (1999) Hepatectomy for hepatocellular carcinoma: toward zero hospital deaths. Ann Surg 229:322-330

3. Imamura H, Seyama Y, Kokudo N et al (2003) One thousand fifty-six hepatectomies without mortality in 8 years. Arch Surg 138:1198-1206

4. Kaibori M, Matsui Y, Kwon AH et al (2005) Prognosis of hepatocellular carcinoma after hepatectomy in patients with renal dysfunction. World J Surg 29:375-381. https://doi.org/10.1007/ s00268-004-7515-0

5. Orii T, Takayama T, Haga I et al (2008) Efficacy of a liver resection for hepatocellular carcinoma in patients with chronic renal failure. Surg Today 38:329-334

6. Toshima T, Shirabe K, Yoshiya S et al (2012) Outcome of hepatectomy for hepatocellular carcinoma in patients with renal dysfunction. HPB (Oxford) 14:317-324

7. Squires MH 3rd, Lad NL, Fisher SB et al (2014) The effect of preoperative renal insufficiency on postoperative outcomes after major hepatectomy: a multi-institutional analysis of 1170 patients. J Am Coll Surg 219:914-922

8. Seyama Y, Kokudo N (2009) Assessment of liver function for safe hepatic resection. Hepatol Res 39:107-116

9. Kidney Disease: Improving Global Outcomes (KDIGO) CKD Work Group (2013) KDIGO clinical practice guideline for the evaluation and management of chronic kidney disease. Kidney Int Suppl 3:1-150

10. Polkinfhorne KR (2011) Controversies in chronic kidney disease staging. Clin Biochem Rev 32:55-59

11. Minutolo R, Lapi F, Chiodini P et al (2014) Risk of ESRD and death in patients with CKD not referred to a nephrologist: a 7-year prospective study. Clin J Am Soc Nephrol 9:1586-1593

12. Lautamaki A, Kiviniemi T, Biancari F et al (2016) Outcome after coronary artery bypass grafting and percutaneous coronary intervention in patients with stage $3 b-5$ chronic kidney disease. Eur J Cardiothorac Surg 49:926-930

13. Llovet JM, Burroughs A, Bruix J (2003) Hepatocellular carcinoma. The Lancet 362:1907-1917

14. Llovet JM, Fuster J, Bruix J et al (2004) The Barcelona approach: diagnosis, staging, and treatment of hepatocellular carcinoma. Liver Transpl 10:115-122

15. Bruix J, Sherman M, Committee Practice Guidelines (2005) American Association for the Study of Liver Diseases. Management of hepatocellular carcinoma. Hepatology 42:1208-1236

16. Dindo D, Demartines N, Clavien PA (2004) Classification of surgical complications. Ann Surg 240:205-213

17. Ishizawa $\mathrm{T}$, Hasegawa $\mathrm{K}$, Kokudo $\mathrm{N}$ et al (2008) Risk factors and management of ascites after liver resection to treat hepatocellular carcinoma. Arch Surg 144:46-51
18. Devins GM, Mendelssohn DC, Barré PE et al (2003) Predialysis psychoeducational intervention and coping styles influence time to dialysis in chronic kidney disease. Am J Kidney Dis 42:693-703

19. Devins GM, Mendelssohn DC, Barre PE et al (2005) Predialysis psychoeducational intervention extends survival in CKD: a 20-year follow-up. Am J Kidney Dis 46:1088-1098

20. Nobili C, Marzano E, Oussoultzoglou E et al (2012) Multivariate analysis of risk factors for pulmonary complications after hepatic resection. Ann Surg 255:540-550

21. Salerno F, Gerbes A, Ginès P et al (2007) Diagnosis, prevention and treatment of hepatorenal syndrome in cirrhosis. Gut $56: 1310-1318$

22. Kokudo T, Hasegawa K, Matsuyama Y et al (2016) Survival benefit of liver resection for hepatocellular carcinoma associated with portal vein invasion. J Hepatol 65:938-943

23. Lovet JM, Fuster J, Bruix J (1999) Intention-to-treat analysis of surgical treatment for early hepatocellular carcinoma: resection versus transplantation. Hepatology 30:1434-1440

24. Poon RT, Fan ST, Lo CM et al (2002) Extended hepatic resection for hepatocellular carcinoma in patients with cirrhosis: is it justified? Ann Surg 236:602-611

25. Cucchetti A, Ercolani G, Vivarelli M et al (2009) Is portal hypertension a contraindication to hepatic resection. Ann Surg 250:922-928

26. Citterio D, Facciorusso A, Sposito C et al (2016) Hierarchic interaction of factors associated with liver decompensation after resection for hepatocellular carcinoma. JAMA Surg 151:846-853

27. Memeo R, de'Angelis N, Compagnon P et al (2014) Laparoscopic vs. open liver resection for hepatocellular carcinoma of cirrhotic liver: a case-control study. World J Surg 38:2919-2926. https://doi.org/10.1007/s00268-014-2659-Z

28. Morise Z, Wakabayashi G (2017) First quarter century of laparoscopic liver resection. World J Gastroenterol 23:3581-3588

29. Guro H, Cho JY, Han HS et al (2018) Outcome of major laparoscopic liver resection for hepatocellular carcinoma. Surg Oncol 27:31-35

30. Cheng SB, Wu CC, Shu KH et al (2001) Liver resection for hepatocellular carcinoma in patients with end-stage renal failure. J Surg Oncol 78:241-246

31. Yeh CN, Lee WC, Chen MF (2005) Hepatic resection for hepatocellular carcinoma in end-stage renal disease patients: two decades of experience at Chang Gung Memorial Hospital. Wourld J Gastroenterol 11:2067-2071

32. Armstrong T, Welsh FK, Wells J et al (2009) The impact of preoperative serum creatinine on short-term outcomes after liver resection. HPB (Oxford) 11:622-628

33. Yeh CC, Lin JT, Jeng LB et al (2013) Hepatic resection for hepatocellular carcinoma patients on hemodialysis for uremia: a nationwide cohort study. World J Surg 37:2402-2409. https://doi. org/10.1007/s00268-013-2137-z 